\title{
Astrophysical detections and databases for the mono deuterated species of acetaldehyde $\mathrm{CH}_{2} \mathrm{DCOH}$ and $\mathrm{CH}_{3} \mathrm{COD} \star$
}

\author{
L. H. Coudert ${ }^{1}$, L. Margulès ${ }^{2}$, C. Vastel ${ }^{3}$, R. Motiyenko ${ }^{2}$, E. Caux ${ }^{3}$, and J.-C. Guillemin ${ }^{4}$ \\ ${ }^{1}$ Institut des Sciences Moléculaires d'Orsay, CNRS, Univ. Paris-Sud, Université Paris-Saclay, 91405 Orsay, France \\ e-mail: laurent.coudert@u-psud.fr \\ ${ }^{2}$ Laboratoire de Physique des Lasers, Atomes et Molécules, UMR CNRS 8523, Bât. P5, Université de Lille I, 59655 Villeneuve \\ d'Ascq Cedex, France \\ 3 IRAP, Université de Toulouse, CNRS, CNES, UPS, 9 Av. Colonel Roche, BP 44346, 31028 Toulouse Cedex 4, France \\ ${ }^{4}$ Univ. Rennes, Ecole Nationale Superieure de Chimie de Rennes, CNRS, ISCR-UMR6226, 35000 Rennes, France
}

Received 11 December 2018 / Accepted 17 February 2019

\begin{abstract}
Context. Detection of deuterated species may provide information on the evolving chemistry in the earliest phases of star-forming regions. For molecules with two isomeric forms of the same isotopic variant, gas-phase and solid-state formation pathways can be differentiated using their abundance ratio.

Aims. Spectroscopic databases for astrophysical purposes are built for the two mono deuterated isomeric species $\mathrm{CH}_{2} \mathrm{DCOH}$ and $\mathrm{CH}_{3} \mathrm{COD}$ of the complex organic molecule acetaldehyde. These databases can be used to search and detect these two species in astrophysical surveys, retrieving their column density and therefore abundances.

Methods. Submillimeter wave and terahertz transitions were measured for mono deuterated acetaldehyde $\mathrm{CH}_{2} \mathrm{DCOH}$ which is a nonrigid species displaying internal rotation of its asymmetrical $\mathrm{CH}_{2} \mathrm{D}$ methyl group. An analysis of a dataset consisting of previously measured microwave data and the newly measured transition was carried out with a model accounting for the large amplitude torsion. Results. The frequencies of 2556 transitions are reproduced with a unitless standard deviation of 2.3 yielding various spectroscopic constants. Spectroscopic databases for astrophysical purposes were built for $\mathrm{CH}_{2} \mathrm{DCOH}$ using the results of the present analysis and for $\mathrm{CH}_{3} \mathrm{COD}$ using the results of a previous spectroscopic investigation. These two species were both searched for and are detected toward a low-mass star-forming region.

Conclusions. We report the first detection of $\mathrm{CH}_{2} \mathrm{DCOH}$ (93 transitions) and the detection of $\mathrm{CH}_{3} \mathrm{COD}$ (43 transitions) species in source B of the IRAS 16293-2422 young stellar binary system located in the $\rho$ Ophiuchus cloud region, using the publicly available ALMA Protostellar Interferometric Line Survey.
\end{abstract}

Key words. astrochemistry - line: identification - ISM: molecules - ISM: abundances

\section{Introduction}

Acetaldehyde and its isotopic species have been the subject of many spectroscopic investigations, due to their astrophysical relevance and to the large amplitude nature of the internal rotation of the methyl group. The microwave spectrum of the normal species was first analyzed by Kilb et al. (1957) and has since then been investigated up to the $v_{\mathrm{t}}=$ 4 torsional state (Herschbach 1959; Iijima \& Tsuchiya 1972; Bauder \& Günthard 1976; Kleiner et al. 1990, 1992, 1996; Smirnov et al. 2014), leading to its detection (Gilmore et al. 1976) in the interstellar medium (ISM). The isotopic species with a symmetrical $\mathrm{CH}_{3}$ or $\mathrm{CD}_{3}$ methyl group were also investigated (Kleiner et al. 1999; Coudert \& López 2006; Elkeurti et al. 2010; Zaleski et al. 2017), but none are detected in the ISM. There is only a limited number of spectroscopic results for isotopic species with a partially deuterated $\mathrm{CH}_{2} \mathrm{D}$ or $\mathrm{CD}_{2} \mathrm{H}$ asymmetrical methyl group. The mono and bideuterated species $\mathrm{CH}_{2} \mathrm{DCOH}$ and $\mathrm{CD}_{2} \mathrm{HCOH}$ have been studied (Turner \& $\mathrm{Cox}$

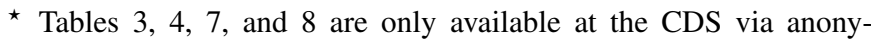
mous ftp to cdsarc.u-strasbg.fr $(130.79 .128 .5)$ or via http: //cdsarc.u-strasbg.fr/viz-bin/qcat?J/A+A/624/A70
1976; Turner et al. 1981), but only a few transitions characterized by a low $K_{\mathrm{a}}$ value were assigned as there was no model available at that time to treat the internal rotation of a partially deuterated methyl group.

Deuterated species are an important tool for understanding interstellar chemistry and specifically surface chemistry (Charnley et al. 1997; Ratajczak et al. 2011). Deuterium forms slightly stronger bonds than hydrogen at low temperatures $(<100 \mathrm{~K})$ and the abundance of deuterium-bearing molecules can become larger than the cosmic $\mathrm{D} / \mathrm{H}$ ratio of $10^{-5}$. A large fractionation ratio has been found in many environments such as dark clouds, low-mass and high-mass protostars, as well as protoplanetary disks (see Ceccarelli et al. 2014, for a review). For complex organic molecules (organic molecules containing at least six atoms, Herbst \& van Dishoeck 2009), there usually exist two different isomeric mono deuterated species and their abundance ratio yields additional information about interstellar chemistry. This may allow differentiation of gas-phase and grain surface formation pathways. For example, the observed gasphase $\left[\mathrm{CH}_{2} \mathrm{DOH}\right] /\left[\mathrm{CH}_{3} \mathrm{OD}\right]$ ratios found in the Orion KL compact ridge as well as the low-mass protostar IRAS 16293-2422, are found to scale inversely with $[\mathrm{HDO}] /\left[\mathrm{H}_{2} \mathrm{O}\right]$ owing to the $\mathrm{H} / \mathrm{D}$ exchange equilibrium between the hydroxyl $(-\mathrm{OH})$ functional 
groups of methanol and water in the ice (Faure et al. 2015). These observations are useful constraints for kinetics models of the deuterium chemistry occurring in the icy mantles of interstellar grains. This article focuses on the study of the mono deuterated $\mathrm{CH}_{2} \mathrm{DCOH}$ and $\mathrm{CH}_{3} \mathrm{COD}$ isotopic variants of acetaldehyde. We present in Sect. 2 the spectroscopic investigation of $\mathrm{CH}_{2} \mathrm{DCOH}$ and the compilation of its database and that of $\mathrm{CH}_{3} \mathrm{COD}$. We first spectroscopically characterized the species that has a partially deuterated $\mathrm{CH}_{2} \mathrm{D}$ methyl group, prior to designing its database. For the species with a symmetrical $\mathrm{CH}_{3}$ methyl group, the database is based on the previous spectroscopic investigation by Elkeurti et al. (2010). We present in Sect. 3 the astrophysical search and detection of both species.

\section{Spectroscopic investigation of $\mathrm{CH}_{2} \mathrm{DCOH}$}

The main isotopic species of acetaldehyde and its isotopic variants with a symmetrical $\mathrm{CH}_{3}$ or $\mathrm{CD}_{3}$ methyl group were studied accounting for their internal rotation with theoretical approaches initially developed for methanol (Koehler \& Dennison 1940; Burkhard \& Dennison 1951; Ivash \& Dennison 1953; Hecht \& Dennison 1957a,b; Lees \& Baker 1968; De Lucia et al. 1989). In the case of the present isotopic species, displaying internal rotation of an asymmetrical partially deuterated $\mathrm{CH}_{2} \mathrm{D}$ methyl group, several theoretical models are also available and were applied to mono and bideuterated methyl formate and methanol (Margulès et al. 2009; Coudert et al. 2012, 2014; Pearson et al. 2012; Ndao et al. 2015).

In this section, the tunneling-rotation energy levels of $\mathrm{CH}_{2} \mathrm{DCOH}$ are calculated using the approach developed for mono deuterated methyl formate (Margulès et al. 2009), based on the high-barrier internal axis method (IAM) approach of Hougen (1985) and Coudert \& Hougen (1988). This IAM treatment is used to analyze the previously available microwave transitions (Turner \& Cox 1976; Turner et al. 1981) and the submillimeter wave and terahertz transitions measured in this work.

\subsection{Experimental}

The transitions measured in this work were recorded in the $150-990 \mathrm{GHz}$ frequency range using the Lille spectrometer (Zakharenko et al. 2015). The absorption cell was a stainlesssteel tube $(6 \mathrm{~cm}$ diameter, $220 \mathrm{~cm}$ long). The sample during measurements was at a pressure of about $10 \mathrm{~Pa}$ and at room temperature; the linewidth was limited by Doppler broadening. The frequency ranges $150-330,400-660$, and 780-990 GHz were covered with various active and passive frequency multipliers from VDI Inc. and an Agilent synthesizer $(12.5-18.25 \mathrm{GHz})$ was used as the source of radiation. Estimated uncertainties for measured line frequencies are either 30 or $50 \mathrm{kHz}$ depending on the observed signal-to-noise ratio $(\mathrm{S} / \mathrm{N})$ and the frequency range. Figure 1 shows two portions of the spectrum recorded in the submillimeter wave region.

\subsection{Theory}

The model developed previously for mono deuterated methyl formate (Margulès et al. 2009) can be applied to mono deuterated acetaldehyde $\mathrm{CH}_{2} \mathrm{DCOH}$ with almost no changes. The coordinates used in this model are the usual Euler angles $\chi, \theta, \phi$ and a large amplitude angular coordinate, denoted $\alpha$, parameterizing the internal rotation of the methyl group with respect to the

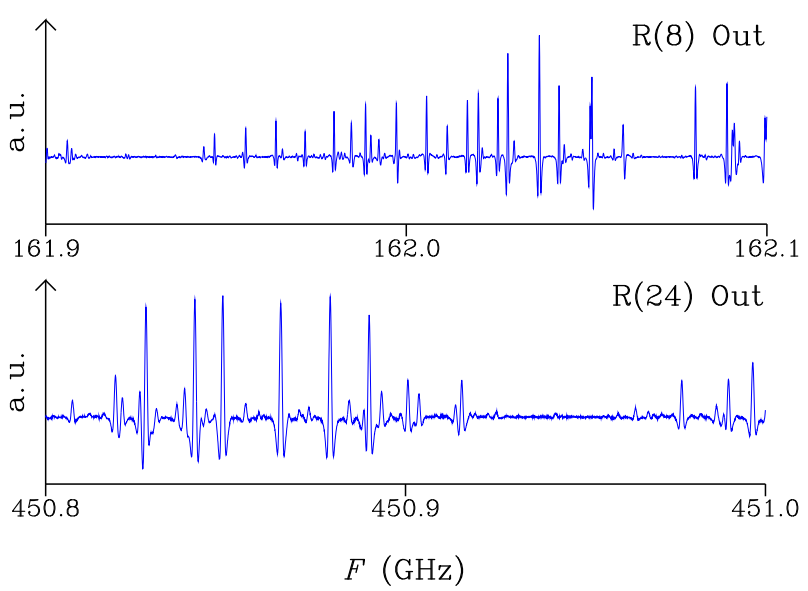

Fig. 1. Two portions of the spectrum recorded in the submillimeter wave region are plotted as a function of the frequency $F$ in GHz. For the Out conformation, the upper and lower panels show tunneling components of the $R(8)$ and $R(24)$ transitions, respectively. The $K$-type structure can be clearly seen in both cases.

aldehyde group. Molecule-fixed coordinates of the atoms are obtained starting from the scheme introduced for the principal axis method in Sect. 3 of Hougen et al. (1994). The initial configuration drawn in their Fig. 1 defines atom positions in an $x^{\prime} y^{\prime} z^{\prime}$ axis system such that the axis of internal rotation coincides with the $z^{\prime}$ axis. The methyl group atoms are numbered from 1 to 3 , with atom 1 being the deuterium atom and atoms 2 and 3, the two hydrogen atoms. The large amplitude coordinate $\alpha$ is the dihedral angle $\angle$ DCCO. Using Sect. 3.1 and Eqs. (1) and (2) of Margulès et al. (2009) allows us to retrieve atom positions in an $x y z$ molecule-fixed axis system which, for any value of $\alpha$, is the principal axis system in the $I^{r}$ representation (Bunker 1979).

In agreement with the IAM approach of Hougen (1985) and Coudert \& Hougen (1988), the non-superimposable equilibrium configurations of the molecule are chosen. There arise three energetically inequivalent configurations, shown in Fig. 2, identified by their configuration number $n$, with $n=1,2$, and 3 , and characterized by $\alpha_{\mathrm{eq}}^{(n)}$ the value of the torsional angle $\alpha$ around which the reference function is centered. Configurations 1 and 2 are the two $C_{1}$ symmetry Out configurations with the deuterium atom outside the $x^{\prime} z^{\prime}$ plane. They are higher in energy than Configuration 3 , the $C_{\mathrm{s}}$ symmetry In configuration with the deuterium atom in the symmetry plane. The energy difference $E_{\mathrm{d}}$ between the zero point energy of the two Out configurations and that of the In configuration was estimated by Turner et al. (1981) to be $15.55 \mathrm{~cm}^{-1}$.

Equations (12) and (13) of Margulès et al. (2009) should be used to obtain the tunneling matrix elements $H_{J K \gamma 1 ; J K^{\prime} \gamma^{\prime} 2}$ of the $1 \rightarrow 2$ tunneling path connecting the isoenergetic Configurations 1 and 2. Similarly, Eqs. (14) and (15) should be used for tunneling matrix elements $H_{J K \gamma 1 ; J K^{\prime} \gamma^{\prime} 3}$ of the $1 \rightarrow 3$ tunneling path connecting Configurations 1 and 3. In Eqs. (12)-(15) of Margulès et al. (2009), $h_{2}$ and $h_{3}$ are the magnitude of the tunneling splittings and $\theta_{2}, \phi_{2}$ and $\chi_{3}, \theta_{3}, \phi_{3}$ are 5 Eulerian-type angles describing the rotational dependence of the tunneling matrix elements. In addition to these parameters, computing the rotationtorsion energy also requires the rotational constants of the In and Out conformations, $A^{\text {In }}, B^{\text {In }}, C^{\text {In }}$ and $A^{\text {Out }}, B^{\text {Out }}, C^{\text {Out }}$, respectively, and their energy difference $E_{\mathrm{d}}$.

When tunneling effects are small, the In conformation displays asymmetric-top rotational energies. For the + and - 

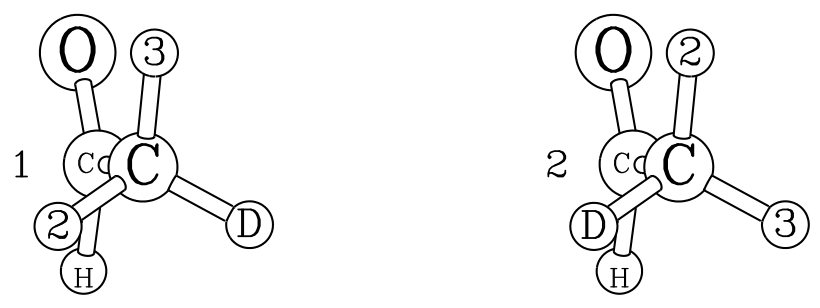

$\alpha_{\mathrm{eq}}^{(1)}=120^{\circ}$

$\alpha_{\mathrm{eq}}^{(2)}=240^{\circ}$

Out

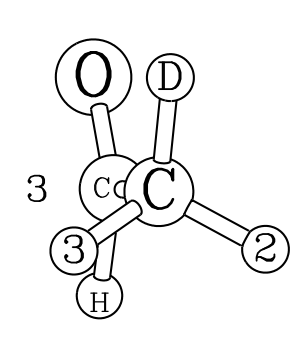

$\alpha_{\text {eq }}^{(3)}=0^{\circ}$ In

Fig. 2. Both energetically equivalent configurations of the Out conformation and the lower energy configuration of the In conformation are identified by their configuration number $n=1,2$, and 3. $\alpha_{\mathrm{eq}}^{(n)}$ is the equilibrium value of the torsional angle $\alpha=\angle \mathrm{DCCO}$. Configuration 3 displays a symmetry plane. Configurations 1 and 2 have $C_{1}$ symmetry.

sublevels arising from the Out conformation, Eq. (21) of Margulès et al. (2009) shows that $E_{\mathrm{d}} \pm h_{2}$ should be added to the asymmetric-top rotational energies, where the upper (lower) sign is for the $+(-)$ sublevel. As $h_{2}$ is negative (Hougen 1985; Coudert \& Hougen 1988), the + sublevel is below the - sublevel. Parallel $a$-type and perpendicular $b$-type transitions arise within the In and Out conformations. For the latter conformation, the selection rule $\pm \leftrightarrow \pm$ holds. Perpendicular $c$-type transitions arise within the Out conformation only and obey the selection rule $\pm \leftrightarrow \mp$.

When tunneling effects are large, distortion terms to the tunneling matrix elements $H_{J K \gamma 1 ; J K^{\prime} \gamma^{\prime} 2}$ and $H_{J K \gamma 1 ; J K^{\prime} \gamma^{\prime} 3}$ should be added and those defined in Eq. (22) of Margulès et al. (2009) are used. Distortion effects to the five Eulerian-type angles are also accounted for using a polynomial-type expansion in $J(J+1)$.

Assigning the levels arising from numerical diagonalization of the Hamiltonian matrix in terms of rotational quantum numbers $K_{a}$ and $K_{c}$ and of + and - tunneling labels is not straightforward as the ordering of the tunneling sublevels changes for large enough $K_{a}$-values. The level assignment chosen here is consistent with symmetry and ensures a smooth variation of the tunneling splitting as a function of $K_{a}$ for each $J$-value. For a given $K_{a}$-value, the tunneling matrix element $H_{J K \gamma 1 ; J K^{\prime} \gamma^{\prime} 2}$ couples the two members of an asymmetry doublet. A mixing of the $J K_{a}, K_{c}, \pm$ and $J K_{a}, K_{c} \pm 1, \mp$ rotational-tunneling sublevels arises and leads to forbidden transitions with even $\Delta K_{a}$ and $\Delta K_{c}$ (Turner et al. 1981). Such transitions were assigned in the previous investigations (Turner \& Cox 1976; Turner et al. 1981) and in the present work.

The Eulerian-type angles $\theta_{2}, \phi_{2}$ and $\chi_{3}, \theta_{3}, \phi_{3}$ were calculated numerically solving Eqs. (49) of Hougen (1985) for each tunneling motion and computing $\alpha$-dependent atom positions with the structure of Kilb et al. (1957). Table 1 lists the computed values along with calculated rotational constants and dipole moment components. The latter were obtained from Turner \& Cox (1978) using their favored orientation.
Table 1. Calculated molecular parameters.

\begin{tabular}{lclc}
\hline \hline Parameter & Value & Parameter & Value \\
\hline$\chi_{2}$ & 249.7 & $\chi_{3}$ & 244.4 \\
$\theta_{2}$ & 2.7 & $\theta_{3}$ & 4.2 \\
$\phi_{2}$ & 69.7 & $\phi_{3}$ & 66.5 \\
\hline$A^{\text {In }}$ & 1.618 & $A^{\text {Out }}$ & 1.742 \\
$B^{\text {In }}$ & 0.329 & $B^{\text {Out }}$ & 0.314 \\
$C^{\text {In }}$ & 0.288 & $C^{\text {Out }}$ & 0.286 \\
\hline$\mu_{x}^{\text {In }}$ & 1.164 & $\mu_{x}^{\text {Out }}$ & 1.054 \\
$\mu_{y}^{\text {In }}$ & - & $\mu_{y}^{\text {Out }}$ & 0.107 \\
$\mu_{z}^{\text {In }}$ & 2.491 & $\mu_{z}^{\text {Out }}$ & 2.538 \\
\hline
\end{tabular}

Notes. Eulerian-type angles, in degrees, involved in the rotational dependence of the tunneling matrix elements, the rotational constants, in $\mathrm{cm}^{-1}$, and the dipole moments components, in Debye, are listed for the In and Out conformations. For symmetry reason, the relation $\chi_{2}=\phi_{2}+\pi$ is fulfilled and $\mu_{y}^{\text {In }}$ is zero. Superscripted In and Out labels identify the rotational constants and dipole moment components.

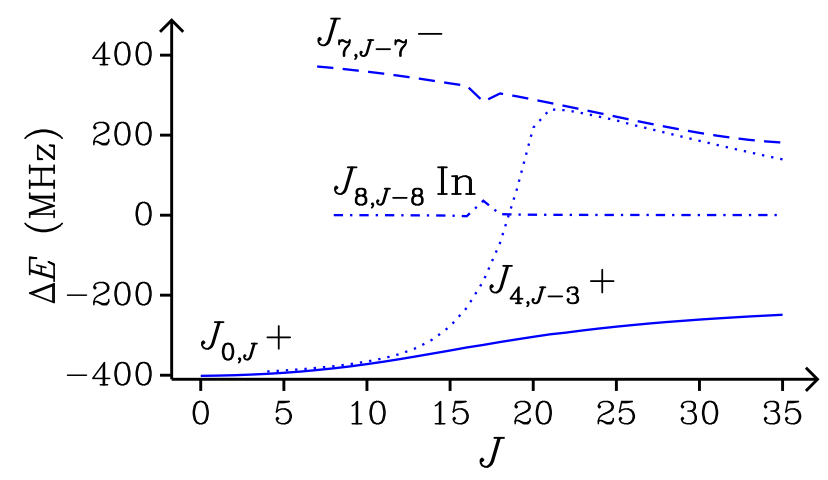

Fig. 3. Effects of the two tunneling motions $\Delta E$ are plotted in $\mathrm{MHz}$ as a function of $J$ for several $K_{a}$-values and for all three tunneling sublevels. In identifies the level arising from the In conformation. + and - identify the tunneling sublevels arising from the Out conformation. The effects of the tunneling motion connecting the In and Out conformations can be seen for the $17_{8,9}$ In and $17_{7,10}$ - sublevels.

\subsection{Line assignment and line frequency analysis}

Starting from the results of Turner \& Cox (1976), parallel $a$ type and perpendicular $b$-type transitions within the In conformation were assigned up to $J=36$ and $K_{a}=3$. This first set of transitions was fitted with a Watson-type Hamiltonian. Parallel $a$-type and perpendicular $b$ - and $c$-type transitions within and between the + and - sublevels of the Out conformation were afterwards assigned up to $J=36$ and $K_{a}=4$, using the results of Turner et al. (1981). Fitting of this second set of transitions yielded rotational constants for the Out conformation, the magnitude of the tunneling splitting $h_{2}$, and the Eulerian-type angles $\theta_{2}$ and $\phi_{2}$. Both sets of transitions were then fitted together and the parameters corresponding to the $1 \rightarrow 3$ tunneling motion and the energy difference $E_{\mathrm{d}}$ could be obtained. Transitions perturbed by the coupling between the In and Out conformations (Cox et al. 2003) could then be included in the fit. New transitions were predicted and searched for. For the In conformation, it was possible to assign $a$-type transitions up to $J=36$ and $K_{a}=14$ and $b$-type transitions up to $J=39$ and $K_{a}=13$. For the Out conformation, $a$-type transitions were assigned up to $J=35$ and $K_{a}=14$ and perpendicular $b$ - and $c$-type transition up to $J=36$ and $K_{a}=5$. The smaller number of perpendicular transitions assigned for the 
Table 2. Assigned transitions.

\begin{tabular}{lcccccc}
\hline & \multicolumn{3}{c}{ In } & \multicolumn{3}{c}{ Out } \\
\cline { 2 - 6 } References & $a$-type & $b$-type & $a$-type & $b$-type & $c$-type & All \\
\hline 1 & 23 & 41 & - & - & - & 64 \\
2 & - & - & 38 & 35 & 21 & 94 \\
This work & 488 & 502 & 1033 & 349 & 26 & 2398 \\
All & 511 & 543 & 1071 & 384 & 47 & 2556 \\
\hline
\end{tabular}

Notes. The number of assigned $a-, b$-, and $c$-type transitions for each conformation in the two previous investigations (Turner \& Cox 1976; Turner et al. 1981) and in this work. $c$-type transitions within the In conformation are not allowed. No transitions were assigned between the In and Out conformations.

Referances. (1) Turner \& Cox (1976); (2) Turner et al. (1981).

Table 5. Spectroscopic parameters

\begin{tabular}{llll}
\hline \hline Parameter & Value & Parameter & Value \\
\hline$A^{\text {In }}$ & $1.6180820(1)$ & $h_{2} \times 10^{3}$ & $-13.3999(7)$ \\
$B^{\text {In }}$ & $0.32905033(1)$ & $\theta_{2}$ & $2.094(7)$ \\
$C^{\text {In }}$ & $0.28810310(1)$ & $\phi_{2}$ & $69.7178(7)$ \\
$E_{d}$ & $15.55866(4)$ & $h_{3} \times 10^{3}$ & $-7.72(7)$ \\
$A^{\text {Out }}$ & $1.745529(1)$ & $\chi_{3}$ & $243.842(140)$ \\
$B^{\text {Out }}$ & $0.31391738(1)$ & $\theta_{3}$ & $3.992(11)$ \\
$C^{\text {Out }}$ & $0.28654111(1)$ & $\phi_{3}$ & $67.021(140)$ \\
\hline
\end{tabular}

Notes. Lowest order parameters obtained in the line position analysis of Sect. 2.3. Parameters are in $\mathrm{cm}^{-1}$ except for the angles $\theta_{2}, \phi_{2}$ and $\chi_{3}, \theta_{3}, \phi_{3}$ which are in degrees. Uncertainties are given in parentheses in the same units as the last quoted digit.

Out conformation than for the In conformation may be due to two factors. The first possible explanation is decreased strength, due to a smaller value of the $x$ dipole moment component of this conformation compared to that of the In conformation, as emphasized by Table 1. Alternatively, there may be a less favorable Boltzmann factor due to a larger $A$ rotational constant for the Out conformation than for the In conformation and the fact that the Out conformation is $15 \mathrm{~cm}^{-1}$ above the In conformation. Table 2 lists the number of assigned transitions for each conformation counting forbidden even $\Delta K_{a}$ and $\Delta K_{c}$ transitions of the Out conformation as $a$-type transitions.

In the final analysis, experimental frequencies were introduced in a least-squares fit procedure where they were given a weight equal to the inverse of their experimental uncertainty squared. Unresolved $K$-type doublets were treated as in Margulès et al. (2009). The rotational Watson-type Hamiltonians used for the In and Out conformations were written using Watson's $A$-set of distortion parameters (Watson 1967, 1968a,b). The root mean square value of the observed minus calculated frequency is $0.054 \mathrm{MHz}$ for transitions within the In conformation, $0.193 \mathrm{MHz}$ for transitions within the Out conformation, and $0.151 \mathrm{MHz}$ for all transitions. The unitless standard deviation of this final analysis is 2.3 For the whole dataset, assignments, observed and calculated frequencies, and residuals are listed in Table 3 available at the CDS. This table displays 13 columns. Columns 1-4 (5-8) give the assignment of the upper (lower) level in terms of $J, K_{a}, K_{c}$ rotational quantum numbers and a vibrational label $v$. The latter is zero for the In conformation and + or - for the two tunneling sublevels of the Out conformation (see Sect. 2.2). Column 9 is the observed frequency
Table 6. Partition function $Q_{\text {rot }}$ of $\mathrm{CH}_{2} \mathrm{DCOH}$ and $\mathrm{CH}_{3} \mathrm{COD}$.

\begin{tabular}{ccc}
\hline & \multicolumn{2}{c}{$Q_{\text {rot }}$} \\
\cline { 2 - 3 }$T / \mathrm{K}$ & $\mathrm{CH}_{2} \mathrm{DCOH}$ & $\mathrm{CH}_{3} \mathrm{COD}$ \\
\hline 2.725 & 12.4 & 25.0 \\
5.000 & 30.8 & 61.0 \\
9.375 & 90.3 & 154.9 \\
18.75 & 343.0 & 435.7 \\
37.50 & 1269.7 & 1234.9 \\
75.00 & 4530.7 & 3740.2 \\
150.0 & 16806.8 & 13769.4 \\
225.0 & 36862.3 & 33051.9 \\
300.0 & 64872.9 & 63715.8 \\
\hline
\end{tabular}

Notes. Partition functions are given for each temperature $T$ in Kelvin.

in $\mathrm{MHz}$; Col. 10 its uncertainty in $\mathrm{kHz}$; Col. 11 is the observed minus calculated residual in $\mathrm{kHz}$; Col. 12 is blank for a single line and $\mathrm{d}$ for a line belonging to an unresolved $K$-type doublet; and Col. 13 gives the reference from which the transition was taken. Table 4, also available at the CDS, lists the parameters determined in the analysis. This table displays 3 columns. Column 1 gives the parameter name; Col. 2 its value; and Col. 3 its uncertainty. Table 5 only lists the lowest order parameters. For the rotational constants, the calculated values in Table 1 are within $0.01 \mathrm{~cm}^{-1}$ from the experimental values in Table 5. For the Eulerian-type angles describing the rotational dependence of the tunneling matrix elements, the discrepancies are at most $5 \%$.

\subsection{Spectroscopic database for $\mathrm{CH}_{2} \mathrm{DCOH}$ and $\mathrm{CH}_{3} \mathrm{COD}$}

For $\mathrm{CH}_{2} \mathrm{DCOH}$, the spectroscopic database was built using the results of the previous sections. Transitions were calculated up to $J=26$ and their line strength and line intensity were computed using the dipole moment components in Table 1. For $\mathrm{CH}_{3} \mathrm{COD}$, the results of the analysis carried out by Elkeurti et al. (2010) were used and transitions were calculated using the same maximum value of $J$. Partition functions $Q_{\text {rot }}$, listed in Table 6, were computed for several temperatures taking a degeneracy factors equal to $(2 J+1)$. A zero energy was taken for the In conformation $0_{00}$ level of $\mathrm{CH}_{2} \mathrm{DCOH}$ and for the $v_{t}=0 A_{1}$ symmetry $0_{00}$ level of $\mathrm{CH}_{3} \mathrm{COD}$. For both species, lines were selected using the procedure in the JPL database catalog line files (Pickett et al. 1998). An intensity cutoff that depended on the line frequency was taken. Its value in $\mathrm{nm}^{2} \cdot \mathrm{MHz}$ units at $300 \mathrm{~K}$ is

$10^{\text {LOGSTR0 }}+(F / 300000)^{2} \times 10^{\text {LOGSTR } 1}$,

where $F$ is the frequency in MHz, and LOGSTR0 and LOGSTR1 are two dimensionless constants both set to -8 . The linelists, given in Table 7 for $\mathrm{CH}_{2} \mathrm{DCOH}$ and in Table 8 for $\mathrm{CH}_{3} \mathrm{COD}$, are available at the CDS. They are formatted in the same way as the catalog line files of the JPL database (Pickett et al. 1998) and display 16 columns. Columns 1-3 contain respectively the line frequency (FREQ) in MHz, the error (ERR) in MHz, and the base 10 logarithm of the line intensity (LGINT) in $\mathrm{nm}^{2} \cdot \mathrm{MHz}$ units at $300 \mathrm{~K}$. Columns 4-6 give the degrees of freedom of the rotational partition function (DR), the lower state energy (ELO) in $\mathrm{cm}^{-1}$, and the upper state degeneracy (GUP), respectively. Columns 7 and 8 contain the species tag (TAG) and format number (QNFMT), respectively. Finally, cols. 9-12 (13-16) give the assignment of the upper (lower) level in terms of $J, K_{a}, K_{c}$, and a vibrational quantum number. For $\mathrm{CH}_{2} \mathrm{DCOH}$, this quantum number is zero for the levels of the In conformation and 1 or 2 for the + and - sublevels of the 
Out conformation. For $\mathrm{CH}_{3} \mathrm{COD}$, this label is 0 for $A$-symmetry levels and 1 and 2 for $E$-symmetry levels when $v_{\mathrm{t}}=0$. This label is 3 and 4 for $E$-symmetry levels and 5 for $A$-symmetry levels when $v_{\mathrm{t}}=1$. For both species, a minimum value of $10 \mathrm{kHz}$ was selected for the calculated error (ERR). For observed unblended microwave lines, the line frequency (FREQ) and the error (ERR) were replaced by their experimental values. This is then indicated by a negative species tag.

\section{Astrophysical observations}

High deuterium fractionation has been observed in various types of environments such as prestellar cores, hot cores, and hot corinos. Its study is considered to be an efficient probe for studying the physical and chemical conditions of these environments and help us to understand their formation. This is especially interesting for the so-called complex organic molecules such as methanol and bigger molecules for which it may allow differentiation of gas-phase and solid-state formation pathways.

We first used the ASAI (Astrochemical Surveys At Iram) ${ }^{1}$ IRAM-30 m Large Program data to search for $\mathrm{CH}_{2} \mathrm{DCOH}$ and $\mathrm{CH}_{3} \mathrm{COD}$. The goal of these observations was to carry out unbiased millimeter line surveys between 80 and $272 \mathrm{GHz}$ of a sample of ten template sources, which fully cover the first stages of the formation process of solar-type stars, from prestellar cores to the late protostellar phase (Lefloch et al. 2018). We used the CASSIS $^{2}$ software for the line identification in the publicly available ASAI data ${ }^{3}$. We conclude that these species are not detected in all ASAI sources with the single-dish observations, either because they are only present in the dense and hot regions as the hot corinos, or present with a too small abundance in the colder extended envelope to be detected by these observations.

We then used ALMA interferometric observations toward the very line rich source IRAS 16293-2422 (hereafter IRAS16293). IRAS16293 is a deeply embedded young stellar binary system located in the L1689 region in the $\rho$ Ophiuchus cloud region, extensively studied through millimeter and submillimeter single-dish and interferometer observations. It has a cold outer envelope (with spatial scales of up to $\sim 6000 \mathrm{au}$ ) (Jaber Al-Edhari et al. 2017) and a hot corino at scales of $\sim 100$ au (Jørgensen et al. 2016). Due to its hot-core-like properties, a wealth of complex organic have been reported toward its two binary components: I16293A and I16293B, separated by $5^{\prime \prime}$ (Wootten 1989). We used the publicly available ALMA Protostellar Interferometric Line Survey (PILS, Jørgensen et al. 2016), an unbiased spectral survey of IRAS 16293 covering a frequency range of about [329-363] GHz of ALMA's Band 7, performed in ALMA's Cycle 2 (project-id: 2013.1.00278.S). Full observational details are given in Jørgensen et al. 2016. The entire raw dataset of this survey is accessible on the ALMA website. In this work, we only used the data obtained with the $12 \mathrm{~m}$ dishes array ( $\sim 38$ antennas in the array at the time of observations), that we reprocessed using the standard pipeline scripts to obtain data-cubes with the ultimate spectral resolution of $\delta v \sim 0.25 \mathrm{~km} \mathrm{~s}^{-1}$, in a $0.5^{\prime \prime}$ beam located $\sim 1^{\prime \prime}$ east of source B $\left(\alpha_{\mathrm{J} 2000}=16 \mathrm{~h} 32 \mathrm{~m} 22.5375 \mathrm{~s} ; \delta_{\mathrm{J} 2000}=-24^{\circ} 28^{\prime} 32.555^{\prime \prime}\right)$, necessary to decrease the damaging effect of line blending.

We first computed local thermodynamic equilibrium (LTE) synthetic spectra, using the CASSIS software, of the expected brightest lines of $\mathrm{CH}_{2} \mathrm{DCOH}$ and $\mathrm{CH}_{3} \mathrm{COD}$ species in the PILS

\footnotetext{
http://www . oan.es/asai/

http://cassis.irap.omp.eu/

http: //WwW . iram. fr/ILPA/LPQ07/
}

frequency range, limiting the search for transitions with $A_{i, j} \geq$ $0.001 \mathrm{~s}^{-1}$ and $E_{\text {up }} \leq 500 \mathrm{~K}$. For the synthetic spectra we assumed a source size larger than the beam $\left(3^{\prime \prime}\right)$, an excitation temperature of $100 \mathrm{~K}$, a line width of $0.8 \mathrm{~km} \mathrm{~s}^{-1}$, and a column density of $5 \times 10^{14} \mathrm{~cm}^{-2}$ for $\mathrm{CH}_{2} \mathrm{DCOH}$ and $3.5 \times 10^{14} \mathrm{~cm}^{-2}$ for $\mathrm{CH}_{3} \mathrm{COD}$ respectively. We limited the search in a $10 \mathrm{GHz}$ spectral band among the $34 \mathrm{GHz}$ of the PILS survey, where the density of $\mathrm{CH}_{2} \mathrm{DCOH}$ and $\mathrm{CH}_{3} \mathrm{COD}$ lines was the largest. The goal being the identification of the $\mathrm{CH}_{2} \mathrm{DCOH}$ and $\mathrm{CH}_{3} \mathrm{COD}$ lines, we did not optimize neither the data processing, nor the CASSIS LTE modeling to reproduce the line intensities. Figures A.1 and A.2 show the detection of $93 \mathrm{CH}_{2} \mathrm{DCOH}$ lines and $43 \mathrm{CH}_{3} \mathrm{COD}$ lines in this $10 \mathrm{GHz}$ frequency range (among, respectively, the 101 and 99 present in the frequency range with the thresholds used). Tables A.1 and A.2 show the detected lines parameters. Note that Jørgensen et al. (2018) reported the detection of $\mathrm{CH}_{3} \mathrm{COD}$ in IRAS 16293-2422B.

It can be noted that with the thresholds used, all $\mathrm{CH}_{2} \mathrm{DCOH}$ lines are detected, except transition $18_{3,16,0}-17_{3,15,0}$, which is predicted to be much brighter than observed and transitions $20_{11,10,1}-19_{11,9,1}, 20_{11,9,1}-19_{11,8,1}, 20_{11,9,2}-19_{11,8,2}$, and $20_{11,10,2}$ $19_{11,9,2}$, which have an $E_{\text {up }} \geq 450 \mathrm{~K}$. For $\mathrm{CH}_{3} \mathrm{COD}$, all undetected transitions but two have an $E_{\text {up }} \geq 340 \mathrm{~K}$. The nondetection of some lines is therefore only due to the sensitivity limit of the PILS survey.

\section{Conclusions}

The rotation-torsion spectrum of the non-rigid mono deuterated acetaldehyde $\mathrm{CH}_{2} \mathrm{DCOH}$ was experimentally and theoretically investigated. Due to the internal rotation of the asymmetrical $\mathrm{CH}_{2} \mathrm{D}$ methyl group, the ground vibrational state of the molecule is split into three torsional sublevels. Transitions within and between these sublevels were measured in the submillimeter wave and terahertz spectra described in Sect. 2.1. These transitions along with previously measured ones (Turner \& Cox 1976; Turner et al. 1981) were fitted using the IAM treatment (Hougen 1985; Coudert \& Hougen 1988) presented in Sect. 2.2. The frequency of 2556 transitions could be reproduced with a 2.3 unitless standard deviation. The good agreement between calculated spectroscopic parameters in Table 1 and their experimental values in Table 5 emphasizes a fairly good understanding of the three first torsional levels of mono deuterated acetaldehyde $\mathrm{CH}_{2} \mathrm{DCOH}$.

The present analysis allowed us to evidence two types of tunneling motions. In addition to the tunneling motion connecting the two energetically equivalent Out configurations, dealt with in mono deuterated methyl formate (Margulès et al. 2009), it was possible to observe the tunneling motion connecting the energetically inequivalent In and Out conformations. This second tunneling motion leads only to shifts as it connects levels that already have different energies (Cox et al. 2003). Figure 3 illustrates the effects of both tunneling motions. The tunneling motion connecting the two energetically equivalent Out configurations leads to an $800 \mathrm{MHz}$ tunneling splitting clearly visible in this figure. The effects of the tunneling motion connecting the In and Out conformations are smaller and become important when level crossings occur.

The results of the analysis were used to build a database for astrophysical purposes for $\mathrm{CH}_{2} \mathrm{DCOH}$. A similar database, for the isomeric mono deuterated species $\mathrm{CH}_{3} \mathrm{COD}$, was compiled starting from the results of the previously published analysis of Elkeurti et al. (2010). With these databases, we have conducted a search of $\mathrm{CH}_{2} \mathrm{DCOH}$ and $\mathrm{CH}_{3} \mathrm{COD}$ lines in the publicly 
available ASAI IRAM-30m Large Program and the ALMA Protostellar Interferometric Line Survey (PILS, Jørgensen et al. 2016). Both $\mathrm{CH}_{2}$ DCOH (93 transitions) and $\mathrm{CH}_{3} \mathrm{COD}$ (43 transitions) species were detected in the IRAS 16293-2422 source B young stellar object alone, located in the $\rho$ Ophiuchus cloud region. Tables A.1 and A.2 list the transitions identified in this source.

\section{References}

Bauder, A., \& Günthard, H. H. 1976, J. Mol. Spectr., 60, 290

Bunker, P. R. 1979, Molecular Symmetry and Spectroscopy, 1st edn. (New York: Academic Press)

Burkhard, D. G., \& Dennison, D. M. 1951, Phys. Rev., 84, 408

Ceccarelli, C., Caselli, P., Bockelée-Morvan, D., et al. 2014, Protostars and Planets VI, 859

Charnley, S. B., Tielens, A. G. G. M., \& Rodgers, S. D. 1997, ApJ, 482, L203

Coudert, L. H., \& Hougen, J. T. 1988, J. Mol. Spectr., 130, 86

Coudert, L. H., \& López, J. C. 2006, J. Mol. Spectr., 239, 135

Coudert, L. H., Margulès, L., Huet, T. R., et al. 2012, A\&A, 543, A46

Coudert, L. H., Zemouli, M., Motiyenko, R. A., Margulès, L., \& Klee, S. 2014, J. Chem. Phys., 140, 064307

Cox, A. P., Hughes, K. H., \& MacDonald, J. N. 2003, Mol. Phys., 101, 569

De Lucia, F. C., Herbst, E., Anderson, T., \& Helminger, P. 1989, J. Mol. Spectr., 134,395

Elkeurti, M., Coudert, L. H., Medvedev, I. R., et al. 2010, J. Mol. Spectr., 263, 145

Faure, A., Faure, M., Theulé, P., Quirico, E., \& Schmitt, B. 2015, A\&A, 584, A98

Gilmore, W., Morris, M., Johnson, D. R., et al. 1976, ApJ, 204, 43

Hecht, K. T., \& Dennison, D. M. 1957a, J. Chem. Phys., 26, 31

Hecht, K. T., \& Dennison, D. M. 1957b, J. Chem. Phys., 26, 48

Herbst, E., \& van Dishoeck, E. F. 2009, ARA\&A, 47, 427

Herschbach, D. R. 1959, J. Chem. Phys., 31, 91

Hougen, J. T. 1985, J. Mol. Spectr., 114, 395

Hougen, J. T., Kleiner, I., \& Godefroid, M. 1994, J. Mol. Spectr., 163, 559

Iijima, T., \& Tsuchiya, S. 1972, J. Mol. Spectr., 44, 88
Ivash, E. V., \& Dennison, D. M. 1953, J. Chem. Phys., 21, 1804

Jaber Al-Edhari, A., Ceccarelli, C., Kahane, C., Viti, S., et al. 2017, A\&A, 597, 40

Jørgensen, J. K., van der Wiel, M. H. D., Coutens, A., et al. 2016, A\&A, 595, A117

Jørgensen, J. K., Müller, H. S. P., Calcutt, H., et al. 2018, A\&A, 620, A170

Kilb, R. W., Lin, C. C., \& Wilson, Jr., E. B. 1957, J. Chem. Phys., 26, 1695

Kleiner, I., Godefroid, M., Herman, M., \& McKellar, A. R. W. 1990, J. Mol. Spectr., 142, 238

Kleiner, I., Hougen, J. T., Suenram, R. D., Lovas, F. J., \& Godefroid, M. 1992, J. Mol. Spectr., 153, 578

Kleiner, I., Hougen, J. T., Grabow, J.-U., et al. 1996, J. Mol. Spectr., 179, 41

Kleiner, I., Lopez, J. C., Blanco, S., McKellar, A. R. W., \& Moazzen-Ahmadi, N. 1999, J. Mol. Spectr., 197, 275

Koehler, J. S., \& Dennison, D. M. 1940, Phys. Rev., 57, 1006

Lees, R. M., \& Baker, J. G. 1968, J. Chem. Phys., 48, 5299

Lefloch, B., Bachiller, R., Ceccarelli, C., et al. 2018, MNRAS, 477, 4792

Margulès, L., Coudert, L. H., Møllendal, H., et al. 2009, J. Mol. Spectr., 254, 55

Ndao, M., Kwabia Tchana, F., Coudert, L. H., et al. 2015, J. Mol. Spectr., 326, 136

Pearson, J. C., Yu, S., \& Drouin, B. J. 2012, J. Mol. Spectr., 280, 119

Pickett, H. M., Poynter, R. L., Cohen, E. A., et al. 1998, J. Quant. Spectr. Radiat. Transfer, 60, 883

Ratajczak, A., Taquet, V., Kahane, C., et al. 2011, A\&A, 528, L13

Smirnov, I. A., Alekseev, E. A., Ilyushin, V. V., et al. 2014, J. Mol. Spectr., 295, 44

Turner, P. H., \& Cox, A. P. 1976, Chem. Phys. Lett., 42, 84

Turner, P. H., \& Cox, A. P. 1978, J. Chem. Soc. Faraday Trans., 2, 533

Turner, P. H., Cox, A. P., \& Hardy, J. A. 1981, J. Chem. Soc. Faraday Trans., 2, 1217

Watson, J. K. G. 1967, J. Chem. Phys., 46, 1935

Watson, J. K. G. 1968a, J. Chem. Phys., 48, 181

Watson, J. K. G. 1968b, J. Chem. Phys., 48, 4517

Wootten, A. 1989, ApJ, 337, 858

Zakharenko, O., Motiyenko, R. A., Margulès, L., \& Huet, T. R. 2015, J. Mol. Spectr., 317, 41

Zaleski, D. P., Duan, C., Carvajal, M., Kleiner, I., \& Prozument, K. 2017, J. Mol. Spectr., 342, 17 


\section{Appendix A: Additional material}
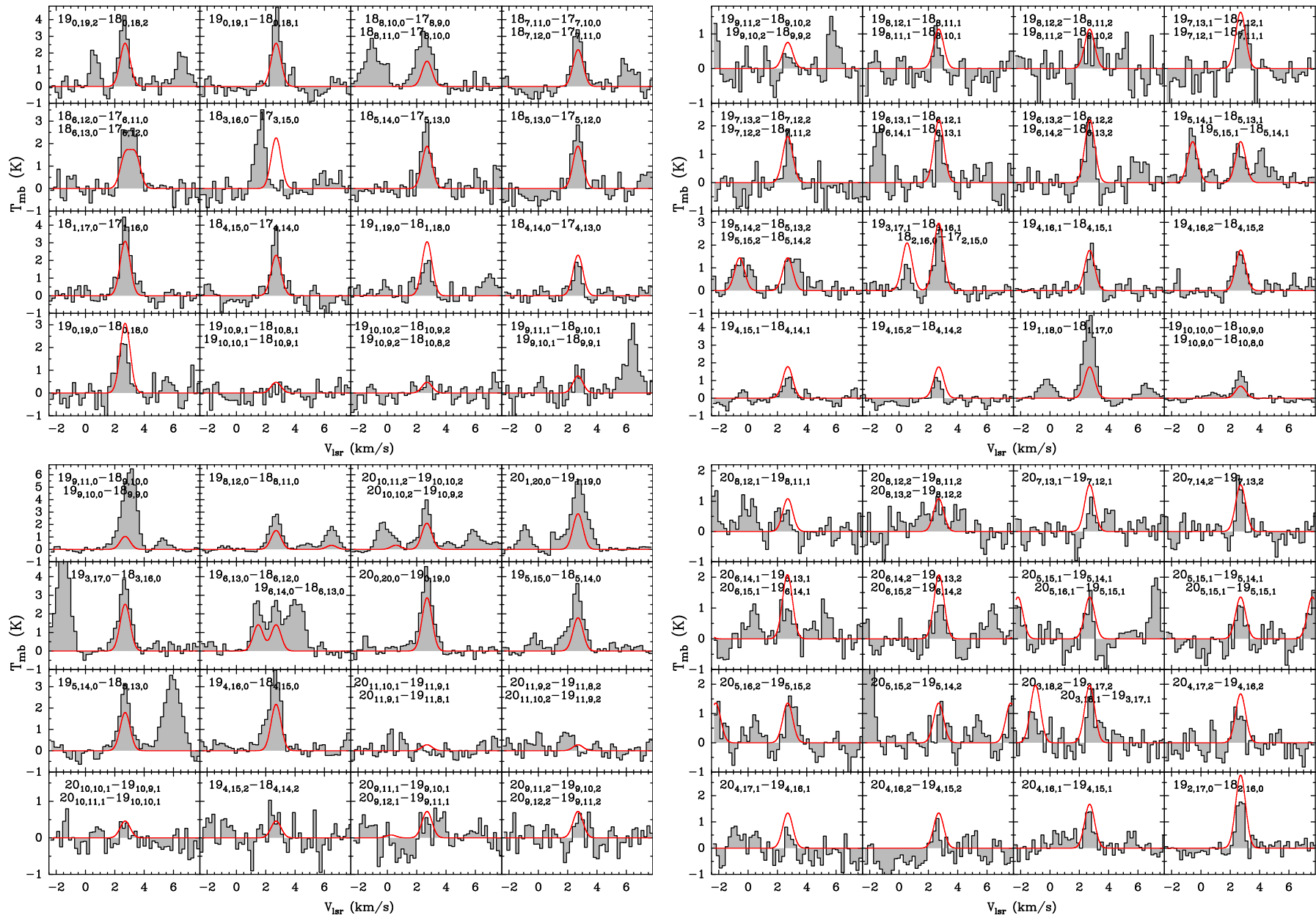

Fig. A.1. $\mathrm{CH}_{2} \mathrm{DCOH}$ observed (in black) and modeled (in red) lines. The lines have been shifted at a $V_{\mathrm{LSR}}$ of $2.7 \mathrm{~km} \mathrm{~s}^{-1}$. The quantum numbers are indicated with a sorting in frequency, for $A_{i, j} \geq 0.001 \mathrm{~s}^{-1}$ and $E_{\text {up }} \leq 500 \mathrm{~K}$. In the case of multiple transitions, the quantum numbers are indicated from the left to the right, with increasing $V_{\mathrm{LSR}} . \mathrm{CH}_{2} \mathrm{DCOH}\left(18_{3,16,0}-17_{3,15,0}\right)$ is not detected, or predicted to be too bright, and the nearby line is $\mathrm{HCOOCH}_{3}$. 

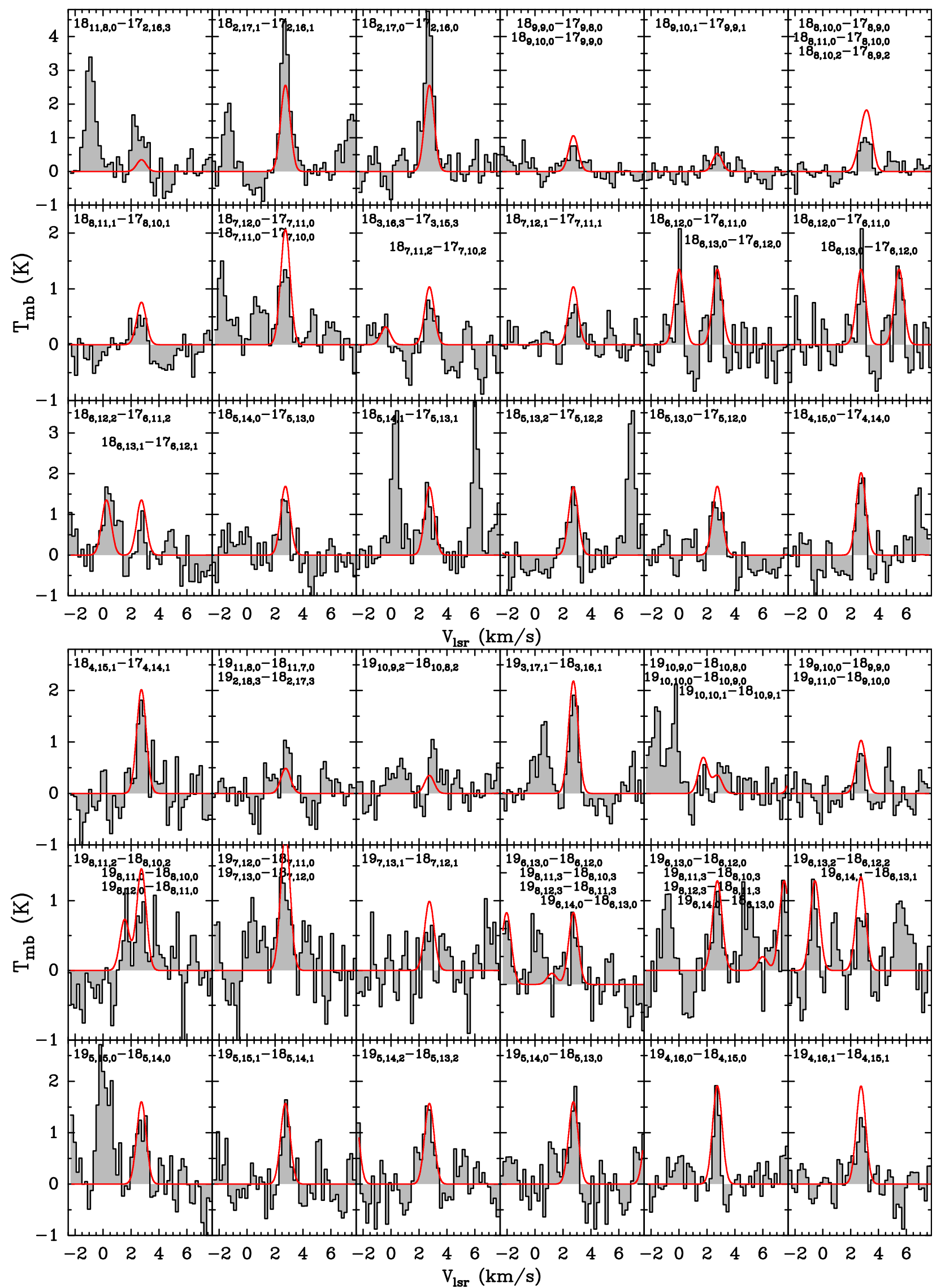

Fig. A.2. $\mathrm{CH}_{3} \mathrm{COD}$ observed (in black) and modeled (in red) lines. The lines have been shifted at a $V_{\mathrm{LSR}}$ of $2.7 \mathrm{~km} \mathrm{~s}^{-1}$. The quantum numbers are indicated with a sorting in frequency, for $A_{i, j} \geq 0.001 \mathrm{~s}^{-1}$ and $E_{\mathrm{up}} \leq 500 \mathrm{~K}$. In the case of multiple transitions, the quantum numbers are indicated from the left to the right, with increasing $V_{\mathrm{LSR}}$. 
Table A.1. $\mathrm{CH}_{2} \mathrm{DCOH}$ transitions in the frequency range considered and their main parameters.

\begin{tabular}{|c|c|c|c|c|c|c|}
\hline Transition & $\begin{array}{c}\text { Frequency } \\
(\mathrm{MHz})\end{array}$ & $\begin{array}{l}E_{\mathrm{up}} \\
(\mathrm{K})\end{array}$ & $\begin{array}{c}A_{i j} \\
10^{-3}\left(\mathrm{~s}^{-1}\right)\end{array}$ & $\begin{array}{c}\tau_{\text {peak }} \\
10^{-2} \\
\end{array}$ & $\int_{\left(\mathrm{K} k \mathrm{~km} \mathrm{~s}^{-1}\right)} T_{\mathrm{mb}} \mathrm{d} v$ & Blend \\
\hline $19_{0,19,2}-18_{0,18,2}$ & 332931.260 & 183.86 & 1.34 & 2.1 & 3.17 & \\
\hline $19_{0,19,1}-18_{0,18,1}$ & 332944.584 & 183.83 & 1.34 & 2.1 & 3.23 & \\
\hline $18_{8,10,0}-17_{8,9,0}$ & 333350.436 & 272.44 & 1.00 & 0.44 & $4.24^{*}$ & Unidentified \\
\hline $18_{8,11,0}-17_{8,10,0}$ & 333350.436 & 272.44 & 1.00 & 0.44 & $4.24^{*}$ & Unidentified \\
\hline $18_{7,12,0}-17_{7,11,0}$ & 333516.699 & 244.24 & 1.11 & 0.714 & 2.94 & \\
\hline $18_{7,11,0}-17_{7,10,0}$ & 333516.714 & 244.24 & 1.11 & 0.714 & 2.94 & \\
\hline $18_{6,13,0}-17_{6,12,0}$ & 333732.064 & 219.82 & 1.16 & 1.04 & 1.64 & \\
\hline $18_{6,12,0}-17_{6,11,0}$ & 333732.844 & 219.82 & 1.16 & 1.04 & 1.64 & \\
\hline $18_{3,16,0}-17_{3,15,0}$ & 333839.703 & 169.17 & 1.07 & 1.58 & ND & \\
\hline $18_{5,14,0}-17_{5,13,0}$ & 334088.542 & 199.20 & 1.21 & 1.44 & 2.35 & \\
\hline $18_{5,13,0}-17_{5,12,0}$ & 334115.850 & 199.20 & 1.21 & 1.25 & 2.34 & \\
\hline $18_{1,17,0}-17_{1,16,0}$ & 334365.794 & 156.58 & 1.30 & 2.78 & 3.81 & \\
\hline $18_{4,15,0}-17_{4,14,0}$ & 334557.044 & 182.39 & 1.25 & 1.57 & 2.59 & \\
\hline $19_{1,19,0}-18_{1,18,0}$ & 334809.383 & 163.53 & 1.32 & 2.68 & 1.88 & \\
\hline $18_{4,14,0}-17_{4,13,0}$ & 335113.524 & 182.46 & 1.26 & 1.57 & 1.20 & \\
\hline $19_{0,19,0}-18_{0,18,0}$ & 335405.895 & 163.43 & 1.32 & 2.17 & 2.18 & \\
\hline $19_{10,10,1}-18_{10,9,1}$ & 341877.416 & 394.14 & 1.06 & 0.088 & 0.13 & \\
\hline $19_{10,9,1}-18_{10,8,1}$ & 341877.416 & 394.14 & 1.06 & 0.088 & 0.13 & \\
\hline $19_{10,10,2}-18_{10,9,2}$ & 341892.791 & 394.12 & 1.06 & 0.088 & 0.32 & \\
\hline $19_{10,9,2}-18_{10,8,2}$ & 341892.791 & 394.12 & 1.06 & 0.088 & 0.32 & \\
\hline $19_{9,10,1}-18_{9,9,1}$ & 341931.674 & 354.75 & 1.13 & 0.162 & 0.70 & \\
\hline $19_{9,11,1}-189,10,1$ & 341931.674 & 354.75 & 1.13 & 0.162 & 0.70 & \\
\hline $19_{9,10,2}-18_{9,9,2}$ & 341947.390 & 354.73 & 1.13 & 0.162 & 0.25 & \\
\hline $19_{9,11,2}-189,10,2$ & 341947.390 & 354.73 & 1.13 & 0.162 & 0.25 & \\
\hline $19_{8,11,1}-18_{8,10,1}$ & 341995.513 & 319.49 & 1.20 & 0.278 & 0.49 & \\
\hline $19_{8,12,1}-18_{8,11,1}$ & 341995.513 & 319.49 & 1.20 & 0.278 & 0.49 & \\
\hline $19_{8,12,2}-18_{8,11,2}$ & 342011.315 & 319.47 & 1.20 & 0.278 & 1.18 & \\
\hline $19_{8,11,2}-18_{8,10,2}$ & 342011.315 & 319.47 & 1.20 & 0.278 & 1.18 & \\
\hline $19_{7,12,1}-18_{7,11,1}$ & 342077.981 & 288.38 & 1.26 & 0.447 & 1.05 & \\
\hline $19_{7,13,1}-18_{7,12,1}$ & 342077.982 & 288.38 & 1.26 & 0.447 & 1.05 & \\
\hline $19_{7,12,2}-18_{7,11,2}$ & 342093.640 & 288.35 & 1.26 & 0.448 & 1.18 & \\
\hline $19_{7,13,2}-18_{7,12,2}$ & 342093.641 & 288.35 & 1.26 & 0.448 & 1.18 & \\
\hline $19_{6,14,1}-18_{6,13,1}$ & 342193.339 & 261.41 & 1.30 & 0.675 & 0.93 & \\
\hline $19_{6,13,1}-18_{6,12,1}$ & 342193.369 & 261.41 & 1.30 & 0.675 & 0.93 & \\
\hline $19_{6,14,2}-18_{6,13,2}$ & 342213.598 & 261.38 & 1.30 & 0.675 & 1.44 & \\
\hline $19_{6,13,2}-18_{6,12,2}$ & 342213.629 & 261.38 & 1.32 & 0.675 & 1.44 & \\
\hline $19_{5,15,1}-18_{5,14,1}$ & 342377.347 & 238.61 & 1.36 & 0.954 & 1.25 & \\
\hline $19_{5,14,1}-18_{5,13,1}$ & 342381.081 & 238.61 & 1.36 & 0.954 & 1.26 & \\
\hline $19_{5,15,2}-18_{5,14,2}$ & 342394.785 & 238.58 & 1.36 & 0.955 & $0.97^{*}$ & $\mathrm{HCOOCH}_{3}$ \\
\hline $19_{5,14,2}-18_{5,13,2}$ & 342398.534 & 238.58 & 1.36 & 0.955 & 1.42 & \\
\hline $18_{2,16,0}-17_{2,15,0}$ & 342601.088 & 162.68 & 1.40 & 2.62 & 1.93 & \\
\hline $19_{3,17,1}-18_{3,16,1}$ & 342603.552 & 205.47 & 1.43 & 1.57 & 0.76 & \\
\hline $19_{3,17,2}-18_{3,16,2}$ & 342607.900 & 205.45 & 1.43 & 1.58 & $?^{*}$ & $\mathrm{CH}_{3} \mathrm{OCH}_{3}$ and $\mathrm{CH}_{3} \mathrm{COD}$ \\
\hline $19_{4,16,1}-18_{4,15,1}$ & 342669.740 & 219.98 & 1.39 & 1.26 & 1.39 & \\
\hline $19_{4,16,2}-18_{4,15,2}$ & 342683.025 & 219.96 & 1.40 & 1.27 & 1.65 & \\
\hline $19_{4,15,1}-18_{4,14,1}$ & 342791.348 & 220.00 & 1.41 & 1.14 & 0.93 & \\
\hline $19_{4,15,2}-18_{4,14,2}$ & 342805.115 & 219.97 & 1.40 & 1.26 & 0.69 & \\
\hline $19_{1,18,0}-18_{1,17,0}$ & 351688.961 & 173.46 & 1.52 & 2.46 & 4.48 & \\
\hline $19_{11,8,0}-18_{11,7,0}$ & 351692.369 & 396.45 & 1.02 & 0.078 & $1.03^{*}$ & $\mathrm{HCOOCH}_{3}$ \\
\hline $19_{11,9,0}-18_{11,8,0}$ & 351692.369 & 396.45 & 1.02 & 0.078 & $1.03^{*}$ & $\mathrm{HCOOCH}_{3}$ \\
\hline $19_{10,10,0}-18_{10,9,0}$ & 351747.890 & 356.99 & 1.11 & 0.145 & 1.27 & \\
\hline $19_{10,9,0}-18_{10,8,0}$ & 351747.890 & 356.99 & 1.11 & 0.145 & 1.27 & \\
\hline $19_{9,10,0}-18_{9,9,0}$ & 351823.926 & 321.28 & 1.19 & 0.254 & $?^{*}$ & $\mathrm{HCOOCH}_{3}$ \\
\hline $19_{9,11,0}-18_{9,10,0}$ & 351823.926 & 321.28 & 1.19 & 0.254 & $?^{*}$ & $\mathrm{HCOOCH}_{3}$ \\
\hline
\end{tabular}

Notes. Integrated intensities noted with a ${ }^{*}$ are certainly blended with lines from other species, indicated in Col. 7 when identified. 
Table A.1. continued.

\begin{tabular}{|c|c|c|c|c|c|c|}
\hline Transition & $\begin{array}{l}\text { Frequency } \\
(\mathrm{MHz})\end{array}$ & $\begin{array}{l}E_{\mathrm{up}} \\
(\mathrm{K})\end{array}$ & $\begin{array}{c}A_{i j} \\
10^{-3}\left(\mathrm{~s}^{-1}\right)\end{array}$ & $\begin{array}{c}\tau_{\text {peak }} \\
10^{-2}\end{array}$ & $\int_{\left(\mathrm{K} \mathrm{km} \mathrm{s}^{-1}\right)} T_{\mathrm{mb}} \mathrm{d} v$ & Blend \\
\hline $19_{8,11,0}-18_{8,10,0}$ & 351931.099 & 289.33 & 1.26 & 0.418 & 2.70 & \\
\hline $19_{8,12,0}-18_{8,11,0}$ & 351931.100 & 289.33 & 1.26 & 0.418 & 2.70 & \\
\hline $19,13,0-187,12,0$ & 352089.199 & 261.14 & 1.33 & 0.646 & 3.40 & \\
\hline $19_{7,12,0}-18_{7,11,0}$ & 352089.229 & 261.14 & 1.33 & 0.646 & 3.40 & \\
\hline $20_{1,20,0}-19_{1,19,0}$ & 352116.723 & 180.43 & 1.53 & 2.37 & 6.58 & \\
\hline $19_{3,17,0}-18_{3,16,0}$ & 352277.871 & 186.08 & 1.50 & 1.72 & 3.79 & \\
\hline $19_{6,14,0}-18_{6,13,0}$ & 352345.833 & 236.73 & 1.39 & 0.942 & 2.57 & \\
\hline $19_{6,13,0}-18_{6,12,0}$ & 352347.267 & 236.73 & 1.39 & 0.942 & 2.16 & \\
\hline $20_{0,20,0}-19_{0,19,0}$ & 352582.537 & 180.36 & 1.54 & 1.98 & 4.23 & \\
\hline $19_{5,15,0}-18_{5,14,0}$ & 352761.312 & 216.13 & 1.44 & 1.29 & 2.84 & \\
\hline $19_{5,14,0}-18_{5,13,0}$ & 352805.826 & 216.13 & 1.44 & 1.16 & 2.71 & \\
\hline $19_{4,16,0}-18_{4,15,0}$ & 353255.649 & 199.34 & 1.48 & 1.67 & 3.22 & \\
\hline $20_{11,10,1}-19_{11,9,1}$ & 359806.816 & 454.93 & 1.19 & 0.041 & ND & \\
\hline $20_{11,9,1}-19_{11,8,1}$ & 359806.816 & 454.93 & 1.19 & 0.041 & ND & \\
\hline $20_{11,10,2}-19_{11,9,2}$ & 359822.499 & 454.91 & 1.19 & 0.041 & ND & \\
\hline $20_{11,9,2}-19_{11,8,2}$ & 359822.499 & 454.91 & 1.19 & 0.041 & ND & \\
\hline $20_{10,10,1}-19_{10,9,1}$ & 359862.321 & 411.42 & 1.28 & 0.08 & 0.15 & \\
\hline $20_{10,11,1}-19_{10,10,1}$ & 359862.321 & 411.42 & 1.28 & 0.08 & 0.15 & \\
\hline $20_{10,11,2}-19_{10,10,2}$ & 359878.052 & 411.39 & 1.28 & 0.08 & 0.58 & \\
\hline $20_{10,10,2}-19_{10,9,2}$ & 359878.052 & 411.39 & 1.28 & 0.08 & 0.58 & \\
\hline $20_{9,11,1}-19_{9,10,1}$ & 359923.175 & 372.03 & 1.36 & 0.146 & 0.33 & \\
\hline $20_{9,12,1}-19_{9,11,1}$ & 359923.175 & 372.03 & 1.36 & 0.146 & 0.33 & \\
\hline $20_{9,12,2}-19_{9,11,2}$ & 359939.252 & 372.00 & 1.36 & 0.146 & 0.39 & \\
\hline $20_{9,11,2}-19_{9,10,2}$ & 359939.252 & 372.00 & 1.36 & 0.146 & 0.39 & \\
\hline $20_{8,13,1}-19_{8,12,1}$ & 359995.506 & 336.77 & 1.43 & 0.25 & 0.36 & \\
\hline $20_{8,12,1}-19_{8,11,1}$ & 359995.506 & 336.77 & 1.43 & 0.25 & 0.36 & \\
\hline $20_{8,12,2}-19_{8,11,2}$ & 360011.751 & 336.74 & 1.43 & 0.25 & 0.97 & \\
\hline $20_{8,13,2}-19_{8,12,2}$ & 360011.751 & 336.74 & 1.43 & 0.25 & 0.97 & \\
\hline $20_{7,14,1}-19_{7,13,1}$ & 360089.336 & 305.66 & 1.50 & 0.4 & 0.49 & \\
\hline $20_{7,13,1}-19_{7,12,1}$ & 360089.337 & 305.66 & 1.50 & 0.4 & 0.49 & \\
\hline $20_{7,14,2}-19_{7,13,2}$ & 360105.836 & 305.63 & 1.50 & 0.4 & 0.93 & \\
\hline $20_{7,13,2}-19_{7,12,2}$ & 360105.837 & 305.63 & 1.50 & 0.4 & 0.93 & \\
\hline $20_{6,15,1}-19_{6,14,1}$ & 360222.466 & 278.70 & 1.56 & 0.6 & 0.71 & \\
\hline $20_{6,14,1}-19_{6,13,1}$ & 360222.514 & 278.70 & 1.56 & 0.6 & 0.71 & \\
\hline $20_{6,15,2}-19_{6,14,2}$ & 360229.586 & 278.67 & 1.55 & 0.598 & 0.86 & \\
\hline $20_{6,14,2}-19_{6,13,2}$ & 360229.635 & 278.67 & 1.55 & 0.598 & 0.86 & \\
\hline $20_{5,16,1}-19_{5,15,1}$ & 360436.463 & 255.90 & 1.61 & 0.845 & 0.75 & \\
\hline $20_{5,15,1}-19_{5,14,1}$ & 360442.349 & 255.91 & 1.61 & 0.845 & 0.68 & \\
\hline $20_{5,16,2}-19_{5,15,2}$ & 360454.387 & 255.88 & 1.61 & 0.846 & 1.33 & \\
\hline $20_{5,15,2}-19_{5,14,2}$ & 360460.297 & 255.88 & 1.61 & 0.846 & 0.71 & \\
\hline $20_{3,18,1}-19_{3,17,1}$ & 360604.606 & 222.77 & 1.68 & 1.39 & 1.10 & \\
\hline $20_{3,18,2}-19_{3,17,2}$ & 360609.057 & 222.76 & 1.68 & 1.39 & 0.72 & \\
\hline $20_{4,17,2}-19_{4,16,2}$ & 360775.557 & 237.27 & 1.65 & 1.05 & 0.90 & \\
\hline $20_{4,17,1}-19_{4,16,1}$ & 360793.435 & 237.30 & 1.32 & 0.894 & 0.31 & \\
\hline $20_{4,16,2}-19_{4,15,2}$ & 360921.148 & 237.29 & 1.32 & 0.837 & 0.45 & \\
\hline $20_{4,16,1}-19_{4,15,1}$ & 360938.372 & 237.32 & 1.65 & 1.12 & 0.97 & \\
\hline $19_{2,17,0}-18_{2,16,0}$ & 361419.301 & 180.02 & 1.64 & 1.93 & 1.25 & \\
\hline
\end{tabular}


Table A.2. $\mathrm{CH}_{3} \mathrm{COD}$ transitions in the frequency range considered and their main parameters.

\begin{tabular}{|c|c|c|c|c|c|c|}
\hline Transition & $\begin{array}{l}\text { Frequency } \\
(\mathrm{MHz})\end{array}$ & $\begin{array}{l}E_{\mathrm{up}} \\
(\mathrm{K})\end{array}$ & $\begin{array}{c}A_{i j} \\
10^{-3}\left(\mathrm{~s}^{-1}\right)\end{array}$ & $\begin{array}{l}\tau_{\text {peak }} \\
10^{-2}\end{array}$ & $\int_{\left(\mathrm{K} \mathrm{km} \mathrm{s}^{-1}\right)} T_{\mathrm{mb}} \mathrm{d} v$ & Blend \\
\hline $18_{11,8,0}-17_{2,16,3}$ & 333512.727 & 361.37 & 1.31 & 0.1916 & $1.05^{*}$ & Unidentified \\
\hline $18_{2,17,1}-17_{2,16,1}$ & 333618.976 & 160.35 & 1.31 & 1.2480 & 3.46 & \\
\hline $18_{2,17,0}-17_{2,16,0}$ & 333645.049 & 160.33 & 1.31 & 1.2470 & 3.66 & \\
\hline $18_{11,8,1}-17_{11,7,1}$ & 334527.523 & 360.87 & 1.31 & 0.1909 & ND & \\
\hline $18_{9,9,2}-17_{9,8,2}$ & 340477.155 & 293.37 & 1.06 & 0.2805 & ND & \\
\hline $189,10,0-17_{9,9,0}$ & 340497.126 & 293.33 & 1.06 & 0.2806 & 0.54 & \\
\hline $189,9,0-17_{9,8,0}$ & 340497.126 & 293.33 & 1.06 & 0.2806 & 0.54 & \\
\hline $18_{9,10,1}-17_{99,1}$ & 340515.997 & 293.32 & 1.06 & 0.2806 & 0.48 & \\
\hline $18_{8,11,0}-17_{8,10,0}$ & 340625.911 & 264.41 & 1.13 & 0.3935 & 0.95 & \\
\hline $18_{8,10,0}-17_{8,9,0}$ & 340625.911 & 264.41 & 1.13 & 0.3935 & 0.95 & \\
\hline $18_{8,10,2}-17_{8,9,2}$ & 340626.479 & 264.42 & 1.13 & 0.3934 & 0.95 & \\
\hline $18_{8,11,1}-17_{8,10,1}$ & 340659.915 & 264.37 & 1.13 & 0.3936 & 0.41 & \\
\hline $18_{3,16,1}-17_{3,15,1}$ & 340701.960 & 170.88 & 1.37 & 1.1410 & 0.89 & \\
\hline $18_{3,16,0}-17_{3,15,0}$ & 340706.208 & 170.88 & 1.37 & 1.1400 & 0.97 & \\
\hline $18_{7,12,0}-17_{7,11,0}$ & 340824.354 & 238.90 & 1.20 & 0.5285 & 1.32 & \\
\hline $18_{7,11,0}-17_{7,10,0}$ & 340824.354 & 238.90 & 1.20 & 0.5285 & 1.32 & \\
\hline $18_{7,11,2}-17_{7,10,2}$ & 340845.770 & 238.89 & 1.20 & 0.5285 & 0.68 & \\
\hline $18_{3,16,3}-17_{3,15,3}$ & 340849.327 & 371.42 & 1.38 & 0.1755 & 0.18 & \\
\hline $18_{7,12,1}-17_{7,11,1}$ & 340864.114 & 238.85 & 1.20 & 0.5287 & 0.62 & \\
\hline $18_{6,13,0}-17_{6,12,0}$ & 341145.085 & 216.82 & 1.26 & 0.6809 & 0.84 & \\
\hline $18_{6,12,0}-17_{6,11,0}$ & 341148.198 & 216.82 & 1.26 & 0.6809 & 0.73 & \\
\hline $18_{6,13,1}-17_{6,12,1}$ & 341180.939 & 216.77 & 1.26 & 0.6811 & 0.44 & \\
\hline $18_{6,12,2}-17_{6,11,2}$ & 341183.787 & 216.78 & 1.26 & 0.6810 & $1.54^{*}$ & $\mathrm{CH}_{3} \mathrm{CHO}$ \\
\hline $18_{12,6,2}-17_{12,5,2}$ & 341334.607 & 399.24 & 1.31 & 0.1287 & ND & \\
\hline $18_{5,14,0}-17_{5,13,0}$ & 341668.402 & 198.18 & 1.31 & 0.8425 & 1.00 & \\
\hline $18_{5,14,1}-17_{5,13,1}$ & 341738.492 & 198.14 & 1.30 & 0.8341 & 0.96 & \\
\hline $18_{5,13,2}-17_{5,12,2}$ & 341745.985 & 198.16 & 1.30 & 0.8339 & 0.96 & \\
\hline $18_{5,13,0}-17_{5,12,0}$ & 341752.782 & 198.20 & 1.32 & 0.8426 & 0.88 & \\
\hline $18_{12,7,1}-17_{126,1}$ & 342038.445 & 398.73 & 1.32 & 0.1290 & ND & \\
\hline $18_{4,15,0}-17_{4,14,0}$ & 342198.102 & 183.00 & 1.36 & 1.0020 & 1.35 & \\
\hline $18_{4,15,1}-17_{4,14,1}$ & 342245.986 & 182.97 & 1.36 & 0.9980 & 1.35 & \\
\hline $18_{4,14,5}-17_{4,13,5}$ & 342379.200 & 384.41 & 1.36 & 0.1525 & ND & \\
\hline $18_{5,14,3}-17_{5,133}$ & 342515.837 & 397.84 & 1.32 & 0.1303 & ND & \\
\hline $18_{5,133}-17_{5,123}$ & 342607.846 & 397.85 & 1.32 & 0.1303 & $?^{*}$ & $\mathrm{CH}_{3} \mathrm{OCH}_{3}$ \\
\hline $18_{3,164}-17_{3,154}$ & 342783.221 & 371.34 & 1.38 & 0.1742 & ND & \\
\hline $18_{4,15,3}-17_{4,14,3}$ & 342789.396 & 383.05 & 1.37 & 0.1545 & ND & \\
\hline $19_{11,9,1}-18_{11,8,1}$ & 352369.864 & 377.78 & 1.53 & 0.1822 & 0.68 & \\
\hline $19_{2,18,4}-18_{2,17,4}$ & 359228.816 & 378.69 & 1.10 & 0.1254 & ND & \\
\hline $19_{11,82}-18_{11,7,2}$ & 359247.409 & 378.67 & 1.10 & 0.1254 & ND & \\
\hline $19_{2,18,3}-18_{2,17,3}$ & 359269.912 & 378.63 & 1.10 & 0.1254 & 0.72 & \\
\hline $19_{11,8,0}-18_{11,7,0}$ & 359269.912 & 378.63 & 1.10 & 0.1254 & 0.72 & \\
\hline $19_{10,9,2}-18_{10,8,2}$ & 359312.215 & 342.96 & 1.20 & 0.1904 & 0.61 & \\
\hline $19_{3,17,1}-18_{3,16,1}$ & 359332.741 & 188.12 & 1.62 & 1.0880 & 1.42 & \\
\hline $19_{3,17,0}-18_{3,16,0}$ & 359340.064 & 188.13 & 1.62 & 1.0880 & 1.15 & \\
\hline $19_{10,10,1}-18_{10,9,1}$ & 359346.965 & 342.92 & 1.20 & 0.1904 & ND & \\
\hline $19_{10,10,0}-18_{10,9,0}$ & 359348.175 & 342.90 & 1.20 & 0.1905 & ND & \\
\hline $19_{10,9,0}-18_{10,8,0}$ & 359348.175 & 342.90 & 1.20 & 0.1905 & ND & \\
\hline
\end{tabular}

Notes. Integrated intensities noted with a ${ }^{*}$ are certainly blended with lines from other species, indicated in Col. 7 when identified. 
A\&A 624, A70 (2019)

Table A.2. continued.

\begin{tabular}{|c|c|c|c|c|c|c|}
\hline Transition & $\begin{array}{c}\text { Frequency } \\
(\mathrm{MHz})\end{array}$ & $\begin{array}{l}E_{\text {up }} \\
(\mathrm{K})\end{array}$ & $\begin{array}{c}A_{i j} \\
10^{-3}\left(\mathrm{~s}^{-1}\right)\end{array}$ & $\begin{array}{c}\tau_{\text {peak }} \\
10^{-2}\end{array}$ & $\int_{\left(\mathrm{K} \mathrm{km} \mathrm{s}^{-1}\right)} T_{\mathrm{mb}} \mathrm{d} v$ & Blend \\
\hline $19_{9,10,2}-18_{9,9,2}$ & 359433.194 & 310.62 & 1.29 & 0.2763 & 0.48 & \\
\hline $19_{9,11,0}-18_{9,10,0}$ & 359453.483 & 310.58 & 1.29 & 0.2764 & 0.50 & \\
\hline $19_{9,10,0}-18_{9,9,0}$ & 359453.483 & 310.58 & 1.29 & 0.2764 & 0.50 & \\
\hline $19_{3,17,3}-18_{3,16,3}$ & 359462.490 & 388.67 & 1.62 & 0.1674 & 0.43 & \\
\hline $19_{9,11,1}-18_{9,10,1}$ & 359473.909 & 310.57 & 1.29 & 0.2764 & 0.35 & \\
\hline $19_{8,12,0}-18_{8,11,0}$ & 359606.525 & 281.67 & 1.37 & 0.3843 & 0.66 & \\
\hline $19_{8,11,0}-18_{8,10,0}$ & 359606.525 & 281.67 & 1.37 & 0.3843 & 0.66 & \\
\hline $19_{8,11,2}-18_{8,10,2}$ & 359607.958 & 281.68 & 1.37 & 0.3842 & 0.40 & \\
\hline $19_{8,12,1}-18_{8,11,1}$ & 359642.724 & 281.63 & 1.37 & 0.3844 & ND & \\
\hline $19_{7,13,0}-18_{7,12,0}$ & 359841.586 & 256.17 & 1.44 & 0.5126 & 1.36 & \\
\hline $19_{7,12,0}-18_{7,11,0}$ & 359841.586 & 256.17 & 1.44 & 0.5126 & 1.36 & \\
\hline $19_{7,122}-18_{7,11,2}$ & 359864.837 & 256.16 & 1.44 & 0.5126 & ND & \\
\hline $19_{7,13,1}-18_{7,12,1}$ & 359883.447 & 256.12 & 1.44 & 0.5128 & 0.37 & \\
\hline $19_{6,14,0}-18_{6,13,0}$ & 360219.543 & 234.11 & 1.51 & 0.6567 & 0.87 & \\
\hline $19_{6,13,0}-18_{6,12,0}$ & 360225.285 & 234.11 & 1.51 & 0.6568 & 0.76 & \\
\hline $19_{6,14,1}-18_{6,13,1}$ & 360258.207 & 234.06 & 1.51 & 0.6570 & 0.67 & \\
\hline $19_{6,13,2}-18_{6,12,2}$ & 360262.184 & 234.07 & 1.51 & 0.6568 & 0.60 & \\
\hline $19_{5,15,0}-18_{5,14,0}$ & 360822.302 & 215.50 & 1.56 & 0.8092 & 1.28 & \\
\hline $19_{5,15,1}-18_{5,14,1}$ & 360895.231 & 215.46 & 1.53 & 0.7938 & 0.95 & \\
\hline $19_{5,14,2}-18_{5,13,2}$ & 360952.814 & 215.48 & 1.54 & 0.7939 & 1.33 & \\
\hline $19_{5,14,0}-18_{5,13,0}$ & 360959.318 & 215.52 & 1.57 & 0.8094 & 1.05 & \\
\hline $19_{3,17,4}-18_{3,16,4}$ & 361083.946 & 388.67 & 1.62 & 0.1661 & ND & \\
\hline $19_{4,16,0}-18_{4,15,0}$ & 361324.526 & 200.34 & 1.61 & 0.9583 & 0.91 & \\
\hline $19_{4,16,1}-18_{4,15,1}$ & 361359.771 & 200.31 & 1.61 & 0.9573 & 1.00 & \\
\hline
\end{tabular}

